\title{
Design of Self-service Car Washing Machine Control System Based on ARM \\ Zhengmin Cui ${ }^{a}$, Peng Sun ${ }^{b}$ \\ Shandong labor vocational and technical college, Jinan, China \\ acuizhengmin888@163.com, b5066408@qq.com
}

Keywords: self-service car washing machine; ARM; 3G network; KINGVIEW6.55, remote monitor

\begin{abstract}
With the cars in our country's rapid increase, the limited quantity of traditional car washing shop has been unable to satisfy people's needs. Responding to this problem the author designed a set of self-service car washing machine control system based on ARM, and a set of remote monitoring system based on 3G mobile network. The terminal control system adopted high speed, low power microcontroller LPC4350 as the control core; adopted RFID read-write module to realize IC card consumption; adopted EM770W 3G module for the washing machine terminal could connect to $3 \mathrm{G}$ mobile network. Through MODBUS protocol, the remote monitoring system based on KINGVIEW6.55 could communicate with the terminal system, and realized the real-time monitoring and recording the washingmachines' working parameters regularly. The system realizes the unattended self-service washing, and greatly reduces the maintenance workload, improve work efficiency.
\end{abstract}

\section{Introduction}

With the rapid development of China's economy and society, people have strong rigid demand of car, and the car ownership continues to show a rapid growth trend. By the end of 2014, the national number of cars rose to 154 million. The limited number of traditional car washing shop has been unable to meet the needs of the majority of owners of car washing. In this paper, we design a self washing machine terminal control system based on LPC4350 micro controller ARM, which is paid by credit card. This car wash way is saving energy, environmental protection, saving land resources, and has the advantages of fast, save time, effort. The computer can connect with the Internet. Through the remote monitoring system based on 3G Network, this system realizes the real-time monitoring and timing of the operation status and fault information of the terminal of the self washing machine. The system without the need for a person on duty, the owner of the vehicle is clean, and the cost is low, the reliability is high, can greatly improve the management of labor intensity and working efficiency.

\section{Design of terminal control system for self washing machine}

The terminal control system structure of the self-help car washing machine is shown in figure 1. System consists of microcontroller LPC4350, dual power supply circuit, keyboard circuit, LCD display circuit, flow meter, liquid level sensor, RFID read and write circuit, relay output circuit, 3G network communication circuit, USB interface circuit and so on. 


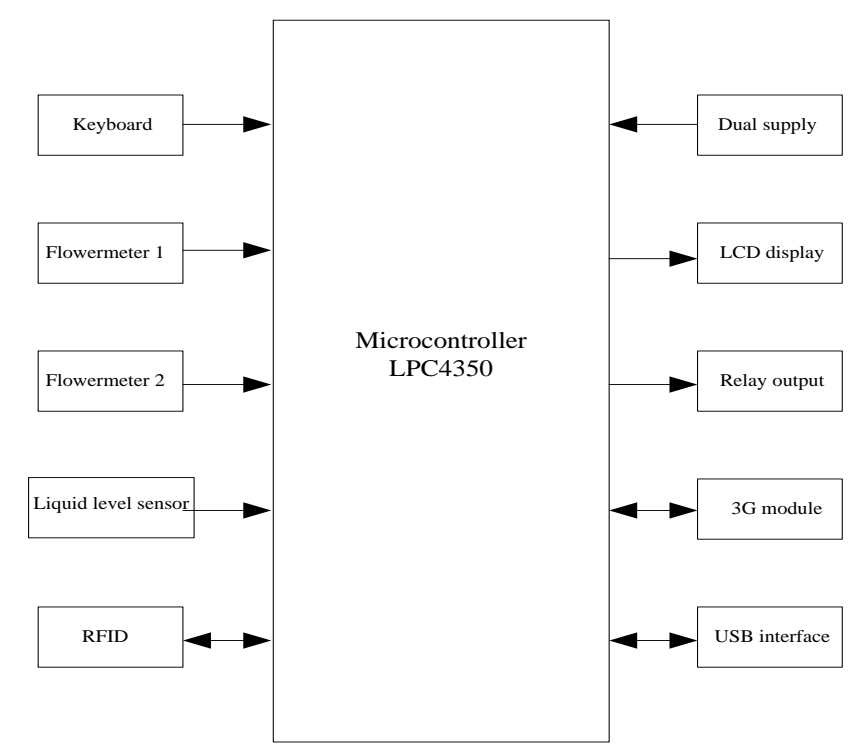

Fig.1 Terminal control system of self-service car washing machine

Control system working principle. Self cleaning machine has water and foam two kinds of washing liquid. When the user swiping IC card, microcontroller will read and write the IC card, and the system will first deduct certain expenses (the cost value can be set). Through pressing water or foam button, users can select the washing liquid, at the same time issue a start command. Having received the start signal, control system will start the washing pump, and open outlet valve of the corresponding (pump or water cannons). During the washing process, control system receives the pulse signal sent from the water and foam flow meter in real time and calculates the amount of pulses. In the course of the car washing, users can pause or switch the washing fluid. After the car washing completed, the user once again swipe the IC card, according to the set of water and foam unit price for billing, system will return the remaining amount to user's IC card.

Liquid level sensor transmits the liquid level signal of water and foam to the micro controller in real time. When the actual level is lower than the setting value, the system can not start the washing process, will issue a warning signal. Through wireless network, EM770W module will transmit the washing machine's work data to the remote monitoring computer. USB interface is used for reading system data and updating system program.

Terminal control system also has the other functions of setting up the system time, the consumption amount of water and foam unit, the total amount of the inquiry, and showing water and foam level.

Microcontroller LPC4350. LPC4350 is launched by NXP for the first time in the world, which uses arm cortex-M4 and Cortex-M0 processor asymmetric dual core digital signal controller. Its working frequency is up to $204 \mathrm{MHz}$, has the advantages of low power consumption, easy to debug, easy integration and so on. It is equipped with up to $1 \mathrm{MB}$ of flash memory, $264 \mathrm{~KB}$ SRAM, integrated high-speed USB, Ethernet, UART, CAN 2.0B, SSP, SPI, I2C serial interface and 164 GPIO, LCD controller, timer/counter, a watchdog timer interface[1]. In the designed control system, LPC4350 plays a role of controlling center. It will collect real-time signal analysis and processing peripherals, responsible for coordinating the operation of the entire control system.

Double power supply circuit. In order to ensure the control system can work normally in the case of a power failure, we designed a dual power supply operating circuit, which is shown in Figure 2. 


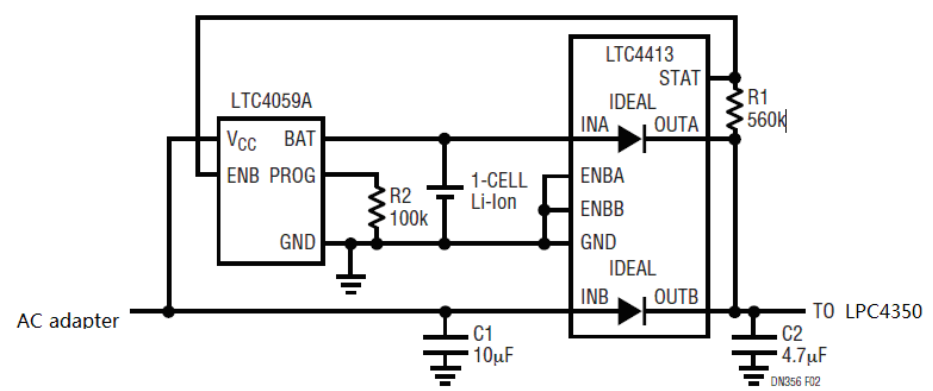

Fig.2 Dual power supply circuit

Dual power supply circuit function is to control the system (or load) of the power supply automatically from the battery to the AC adapter, and control a LTC4059A battery charger. When the AC adapter is not connected, the LTC4413 will load the load connected to the Li ion battery, the STAT pin is high, and the battery charger failure. If connected to the AC adapter, the load voltage will rise with the connection between the INB and the OUTB. When the load voltage exceeds the battery voltage, the battery will be immediately connected to the load, and the STAT pin voltage drops, so as to connect the LTC4059A battery charger and start a charging cycle. When the AC adapter is removed, the load voltage will drop sharply, when the battery voltage falls below the battery will resume to the load supply, the STAT pin voltage drops, which leads to the battery charger failure.

Double power supply circuit switching time is less than $0.1 \mathrm{~ms}$. The circuit also has a limited flow and thermal protection, and the protection of the device from the voltage spike and the slow off function, and when the AC power supply to the load, can replace the battery, and will not interfere with power output. Therefore, the circuit can realize the control system and improve the reliability of the control system [2].

Flow sensor. Water and foam flow sensor using SEN-HZW3WA pulse signal flow sensor. The sensor loads resistance into the positive pole of Hall element, and through the 5V DC voltage to make the current direction and magnetic field direction orthogonal. When the water through the turbine switch shell to drive the magnetic rotor rotation, the rotor produces different magnetic poles of the rotating magnetic field, and cuts the magnetic induction line, then produces high and low pulse level. The output pulse signal frequency of Hall element is proportional to the speed of the magnetic rotor, and the rotor speed is proportional to the water flow rate ${ }^{[3]}$. The formula for pulse signal and water instantaneous flow is: $\mathrm{F}=8.1 \mathrm{Q}-3$.

In the formula, $\mathrm{F}$ is the pulse signal frequency, $\mathrm{Q}$ is the water flow.

The conversion can be obtained that when the sensor accumulated a total of 477 pulses, equivalent to the flow through $1 \mathrm{~L}$ water. In this system, the micro controller LPC4350 calculates the cumulative flow rate of the car wash by capturing the number of pulses emitted by the two sensors.

\section{G network connection}

Control system uses EM770W module to access the 3G network of the self-service car washing machine. EM770W is the production of HUAWEI 3G wireless communication module, which can support WCDMA, and widely used in the terminal equipment and industrial fields; in high speed packet access technology (HSPA), its uplink download rate is $7.2 \mathrm{Mbit} / \mathrm{s}$, the downlink download rate is $5.76 \mathrm{Mbit} / \mathrm{s}$. It has the advantages of low power consumption, good compatibility, high transmission speed, strong stability and other characteristics [4]. EM770W has two UART interfaces, the UART1 can support flow control function, PPP dialing, sending AT commands. In the control system, the UART1 interface of the micro controller LPC4350 is connected with the UART1 to realize the data transmission with the remote monitoring computer.

Using the 3G network module, the self-service car washing machine terminal and the remote monitoring computer can carry out the transparent data communication. That is to say, there is no communication protocol between the car washing machine terminal MCU and 3G network module, the 3G network module only sends the data that MCU sends over to go out, and the software can 
receive the data packets from the $3 G$ module [5]. Such as car wash machine terminal MCU sends a byte data to $0 x F F$, then the software will receive a byte data on the remote computer $0 x F F$. The communication principle of the data from the remote computer to the washing machine is the same.

\section{Software design of remote monitoring computer}

Through 3G mobile communication network, the car washing machine terminal control system upload work data to the remote server, the server receives the remote data and saves it to the database.

To facilitate the management of remote view the washing machine terminal, we use Kingview 6.55 platform to compile a set of computer remote monitoring software. Kingview 6.55 supports industrial real-time database, with huge data storage capacity, has an open structure, can with a variety of communication protocols interconnect (such as MODBUS, PPI Protocol), and the integration of a variety of drivers, and a variety of hardware manufacturers of $\mathrm{I} / \mathrm{O}$ device communication [6].

The function of remote computer monitoring software is as follows.

- Parameter setting and status display. The administrator can view the communication state of the terminal, the amount of water and foam, the total amount of the user's credit card, and can set the system time, unit price, liquid level and so on.

- Real-time alarm. Monitoring system can real-time monitor the level of the car washing machine terminal, communication status, and so on. When an alarm event occurs, the system will show the alarm events in alarm display window, and record the data in alarm information database, for the management of personnel to inspect and reference.

- The database storage system can complete the real time data processing, historical data storage and data report. Computer record the car washing machine terminal work data on time. Using the embedded report system and the rich report function, the software can realize various operations, data conversion, statistical analysis and report printing, and overcome the tedious and random ${ }^{[7]}$.

\section{Conclusion}

The design of self - car washing control system based on ARM reflects three major advantages. First, self control water consumption, saving cost. According to statistics, the washing water consumption is about 20L, only $1 / 3$ of the car washing shop, by rough estimate, which can save about 1 billion cubic meters water. Second, it has the functions of water washing and foam cleaning. It is easy to switch, low cost, and the average price of a car wash at around 5 yuan. Third, the system can be $24 \mathrm{~h}$ on work, without the need for a person on duty.

Through the remote computer monitoring system based on 3G network, administrator can access the Internet to the computer, on this computer administrator can see the work state of the car washing machines. The designed system has been put into operation, its performance is stable, and the data transmission is good, the speed is fast, the scope of monitoring is not limited. So it greatly reduces the workload of the car washing machine, and improves the working efficiency.

\section{References}

[1] Zhang C F, Sprinter introduced dual channel ideal diode LTC4413. Electronic Test, 2005, (1):31-32

[2] Ye Y H, Ye L R. Application of Water F low Sensor to Gas Water Heater. Gas and Heat, 2005, 25(10):22 23

[3] Li W S. Design of Wireless Video Surveillance Based on 3G. Tianjin: Tianjin University, 2011

[4] Chen W B, Liu G C, Feng L. Design of Positioning and Video Surveillance System for Vehicles Based on 3G. Computer Measurement and Control 2011, 19(3):600-602 
[5] Yuan S, Huang H S. Design Study on Construction Machinery Electrical Controller Based on MCU and $\mu \mathrm{C} / \mathrm{OS}-\mathrm{II}$. Measurement and Control Technology, 2014, 33(4):58-61

[6] $\mathrm{Hu}$ X G. Application of Electromagnetic Flowmeter in Reservoir Culvert Flow Measuring Based on Modbus Protocol. Measurement and Control Technology, 2014, 33(9):101-104

[7] Cheng J H, Tang C L, Huang Y R, etal. Design and Implementation of Ventilators Monitoring System Based on Zigbee and Kingview. Coal Mine Machinery, 2014, 35(8):243-245 